Supporting Information for:

\title{
Three-Coordinate Iron(II) Expanded Ring N-Heterocyclic Carbene Complexes
}

Jay J. Dunsford, ${ }^{\dagger}$ David J. Evans, ${ }^{\ddagger}$ Thomas Pugh, ${ }^{\dagger}$ Sachin N. Shah, ${ }^{\ddagger}$ Nicholas F. Chilton, ${ }^{*}, \dagger$ and Michael J. Ingleson*,†

\footnotetext{
${ }^{\dagger}$ School of Chemistry, University of Manchester, Brunswick Street, M13 9PL, UK.

* Department of Chemistry, University of Hull, Cottingham Road, Hull, HU6 7RX, UK.
}

\section{Table of Contents}

1. X-Ray Crystallography $\quad$ S2

2. Mössbauer Spectroscopy Data $\quad$ S4

3. SQUID Magnetometry Data S6

4. CASSCF Calculation Information S6

$\begin{array}{ll}\text { 4. References } & \text { S7 }\end{array}$

5. NMR Data for All Novel Compounds $\quad$ S8

6. Additional NMR Experiments $\quad$ S11 


\section{X-Ray Crystallography}

Data for compounds 2. $\mathbf{C}_{6} \mathbf{D}_{6}, \mathbf{2 .} \mathbf{C}_{7} \mathbf{H}_{8}, 3, \mathbf{3}, \mathbf{5}$ and $\mathbf{6}$ were recorded on an Agilent Supernova diffractometer, with Mo $\mathrm{K} \alpha$ radiation (mirror monochromator, $\lambda=0.7107$ ). The CrysAlisPro $^{[1]}$ software package was used for data collection, cell refinement and data reduction. Data for 1 was recorded on a Bruker APEX-II diffractometer, with $\mathrm{Cu} \mathrm{K \alpha}$ radiation (graphite monochromator, $\lambda=1.5418 \AA$ ). The Bruker APEX2 software package was used for data collection and the CrysAlisPro ${ }^{[1]}$ software package was used for cell refinement and data reduction. For all data sets the CrysAlisPro software package was used for empirical absorption corrections, which were applied using spherical harmonics, implemented in SCALE3 ABSPACK scaling algorithm. All structures were solved using direct methods and refined against F2 using the OLEX2 ${ }^{[2]}$ software package. Non-hydrogen atoms were refined anisotropically. Hydrogen atoms were all located in a difference map and repositioned geometrically.

Crystallographic data have been deposited with the Cambridge Crystallographic Data Centre under references: 1435131, 1446871, 1446872, 1435128, 1435129, 1446021, and 1435130 for $1,2 . C_{6} D_{6}, 2 . C_{7} H_{8}, 3,4,5$ and 6 , respectively. These data can be obtained free of charge from The Cambridge Crystallographic Data Centre via www.ccdc.cam.ac.uk/data_request/cif.

Table S1. Crystal Structure Refinement Data for $\mathbf{1}, \mathbf{2 .} \mathbf{C}_{6} \mathbf{D}_{\mathbf{6}}$ and $\mathbf{2 .} \mathbf{C}_{7} \mathbf{H}_{\mathbf{8}}$

\begin{tabular}{|c|c|c|c|}
\hline & $\mathbf{1}$ & $2 . C_{6} D_{6}$ & $2 . \mathrm{C}_{7} \mathrm{H}_{8}$ \\
\hline Empirical Formula & $\mathrm{C}_{29} \mathrm{H}_{42} \mathrm{Br}_{2} \mathrm{FeN}_{2}$ & $\mathrm{C}_{35} \mathrm{H}_{48} \mathrm{Cl}_{2} \mathrm{FeN}_{2}$ & $\mathrm{C}_{32.5} \mathrm{H}_{42} \mathrm{Cl}_{2} \mathrm{FeN}_{2}$ \\
\hline Fw/g mol ${ }^{-1}$ & 634.31 & 623.50 & 587.43 \\
\hline Cryst syst, Space Group & Monoclinic, $\mathrm{P} 21 / \mathrm{n}$ & Monoclinic, $\mathrm{P} 2_{1} / \mathrm{c}$ & Monoclinic, $\mathrm{P} 2{ }_{1} / \mathrm{c}$ \\
\hline $\mathbf{a} / \AA$ & $9.43883(12)$ & $17.3888(11)$ & $11.155(2)$ \\
\hline b/Å & $24.9246(3)$ & $9.6923(4)$ & $19.056(4)$ \\
\hline c/ $\AA$ & $12.43441(14)$ & $21.2098(12)$ & $15.342(3)$ \\
\hline$\alpha /$ deg & 90 & 90 & 90 \\
\hline$\beta /$ deg & $95.9310(11)$ & $112.052(7)$ & $96.520(9)$ \\
\hline$\gamma / \mathrm{deg}$ & 90 & 90 & 90 \\
\hline Vol/ $/ \AA^{3}$ & $2909.64(6)$ & $3313.1(3)$ & $3240.2(11)$ \\
\hline $\mathrm{Z}$, calc density $\left(\mathrm{Mg} \mathrm{m}^{-3}\right)$ & $4,1.448$ & $4,1.250$ & $4,1.204$ \\
\hline $\operatorname{abs} \operatorname{coeff}\left(\mathrm{mm}^{-1}\right)$ & 7.503 & 0.642 & 0.652 \\
\hline $\mathbf{F}(000)$ & 1304.0 & 1328.0 & 1240.0 \\
\hline cryst, colour & Plate, Colourless & Block, colourless & Block, colourless \\
\hline cryst dimens $/ \mathrm{mm}^{3}$ & $0.2 \times 0.2 \times 0.1$ & $0.4 \times 0.4 \times 0.2$ & $0.4 \times 0.3 \times 0.2$ \\
\hline$\theta$ range (deg) & 7.094 to 133.19 & 6.576 to 50.048 & 6.058 to 50.054 \\
\hline Reflns collected /unique & $35861 / 5080$ & $12492 / 5844$ & $17089 / 5688$ \\
\hline $\mathbf{R}_{\text {int }}$ & 0.0259 & 0.0295 & 0.0774 \\
\hline Data/restraints/ parameters & $5080 / 0 / 315$ & 5844 / 0 / 369 & 5688 / 0 / 360 \\
\hline final $R$ indicies $\left[F^{2}<2 \theta\left(F^{2}\right)\right]:$ & $0.0204,0.0503$ & $0.0498,0.1043$ & $0.0802,0.1486$ \\
\hline $\mathbf{R}$ indicies (all data): $\mathbf{R} 1$, wR2 & $0.0211,0.0506$ & $0.0795,0.1143$ & $0.1497,0.1864$ \\
\hline Largest diff peak and hole/e $\AA^{-3}$ & $0.30 /-0.45$ & $0.41 /-0.40$ & $0.51 /-0.58$ \\
\hline
\end{tabular}


Table S2. Crystal Structure Refinement Data for 3, 4, 5 and 6

\begin{tabular}{|c|c|c|c|c|}
\hline & 3 & 4 & 5 & $\overline{6}$ \\
\hline $\begin{array}{l}\text { Empirical Formula } \\
\text { Fw/g mol }{ }^{-1}\end{array}$ & $\begin{array}{l}\mathrm{C}_{27} \mathrm{H}_{38} \mathrm{Br}_{2} \mathrm{FeN}_{2} \mathrm{O} \\
622.26\end{array}$ & $\begin{array}{l}\mathrm{C}_{35} \mathrm{H}_{42} \mathrm{Br}_{2} \mathrm{FeN}_{2} \cdot 2 \mathrm{C}_{6} \mathrm{H}_{6} \\
706.37\end{array}$ & $\begin{array}{l}\mathrm{C}_{78} \mathrm{H}_{100} \mathrm{Br}_{4} \mathrm{Fe}_{2} \mathrm{~N}_{4} \\
1524.95\end{array}$ & $\begin{array}{l}\mathrm{C}_{31} \mathrm{H}_{46} \mathrm{Br}_{2} \mathrm{FeN}_{2} \mathrm{O} \\
678.37\end{array}$ \\
\hline $\begin{array}{l}\text { Cryst syst, space } \\
\text { Group }\end{array}$ & Triclinic, $\mathrm{P}-1$ & Monoclinic, P21/c & Triclinic, P-1 & $\begin{array}{l}\text { Orthorhombic, } \\
\text { Pbca }\end{array}$ \\
\hline $\mathbf{a} / \mathbf{A}$ & $8.4392(4)$ & $12.2689(4)$ & $10.6547(5)$ & $15.5289(4)$ \\
\hline $\mathbf{b} / \mathbf{A}$ & $12.4618(5)$ & $16.2289(3)$ & $12.2902(8)$ & $18.4203(6)$ \\
\hline $\mathbf{c} / \AA ̊ \AA$ & $13.7286(6)$ & $18.0297(5)$ & $16.2704(13)$ & $22.0688(6)$ \\
\hline$\alpha / \operatorname{deg}$ & $78.655(4)$ & 90 & $70.448(7)$ & 90 \\
\hline$\beta / \operatorname{deg}$ & $80.812(4)$ & $108.813(3)$ & $88.517(5)$ & 90 \\
\hline$\gamma / \mathrm{deg}$ & $80.569(4)$ & 90 & $67.228(5)$ & 90 \\
\hline $\mathrm{Vol} / \AA^{3}$ & $1384.29(11)$ & $3398.10(17)$ & $1838.0(2)$ & $6312.7(3)$ \\
\hline $\begin{array}{l}\text { Z, calc density (Mg m- } \\
\text { 3) }\end{array}$ & $2,1.493$ & $4,1.381$ & $2,1.378$ & $8,1.428$ \\
\hline abs coeff $\left(\mathbf{m m}^{-1}\right)$ & 3.452 & 2.820 & 2.612 & 3.035 \\
\hline $\mathbf{F}(\mathbf{0 0 0})$ & 636.0 & 1448.0 & 788.0 & 2800.0 \\
\hline cryst, colour & Block, Colourless & Block, Colourless & $\begin{array}{l}\text { Block, } \\
\text { Colourless }\end{array}$ & Block, Colourless \\
\hline cryst dimens $/ \mathrm{mm}^{3}$ & $0.4 \times 0.3 \times 0.3$ & $0.3 \times 0.3 \times 0.2$ & $0.7 \times 0.3 \times 0.2$ & $0.5 \times 0.2 \times 0.2$ \\
\hline$\theta$ range (deg) & 6.732 to 58.058 & 6.776 to 50.052 & 6.716 to 52.744 & 6.788 to 50.048 \\
\hline $\begin{array}{l}\text { Reflns collected / } \\
\text { unique }\end{array}$ & 11179 / 6298 & $13336 / 5989$ & $13192 / 7489$ & $15419 / 5552$ \\
\hline $\mathbf{R}_{\text {int }}$ & 0.0211 & 0.0195 & 0.0361 & 0.0274 \\
\hline $\begin{array}{l}\text { Data/restraints/ } \\
\text { parameters }\end{array}$ & 6298 / 0 / 304 & $5989 / 0 / 429$ & 7489 / 0 / 393 & $5552 / 0 / 342$ \\
\hline $\begin{array}{l}\text { final } R \text { indicies }\left[F^{2}<\right. \\
\left.2 \theta\left(F^{2}\right)\right]:\end{array}$ & $0.0318,0.0692$ & $0.0331,0.0862$ & $0.0537,0.1193$ & $0.0307,0.0631$ \\
\hline $\begin{array}{l}\mathbf{R} \text { indicies (all data): } \\
\mathbf{R} 1, \mathbf{w R 2}\end{array}$ & $0.0409,0.0730$ & $0.0423,0.0908$ & $0.0812,0.1336$ & $0.0432,0.0677$ \\
\hline $\begin{array}{l}\text { Largest diff peak and } \\
\text { hole/e } \AA^{-3}\end{array}$ & $0.67 /-0.78$ & $0.36 /-0.36$ & $1.08 /-0.83$ & $0.59 /-0.59$ \\
\hline
\end{tabular}




\section{Mössbauer Spectroscopy Data}

100.6

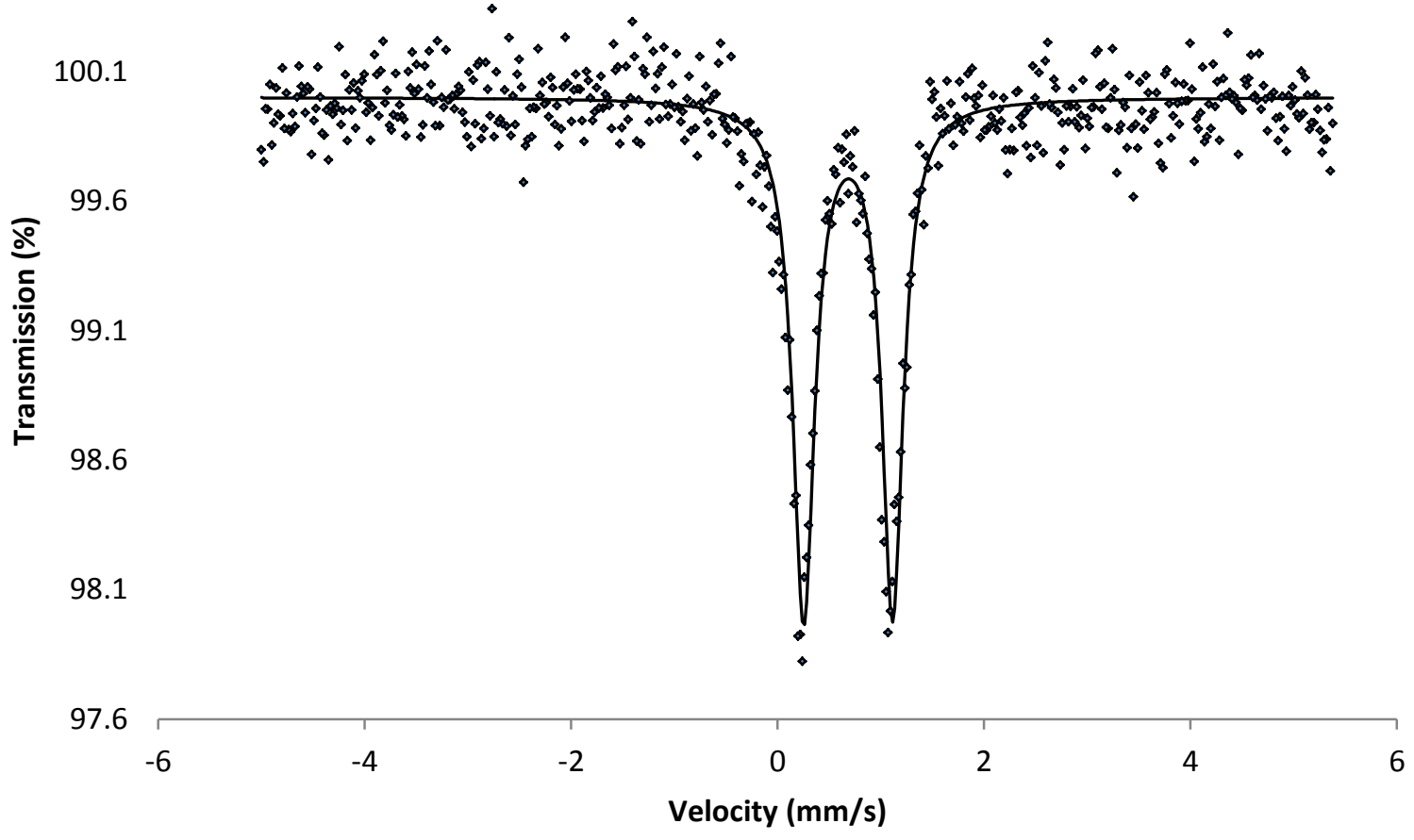

Figure S1. Mössbauer spectra of [Fe(7-DiPP) $\left.\mathrm{Br}_{2}\right], \mathbf{1}$ recorded at $80 \mathrm{~K}$.

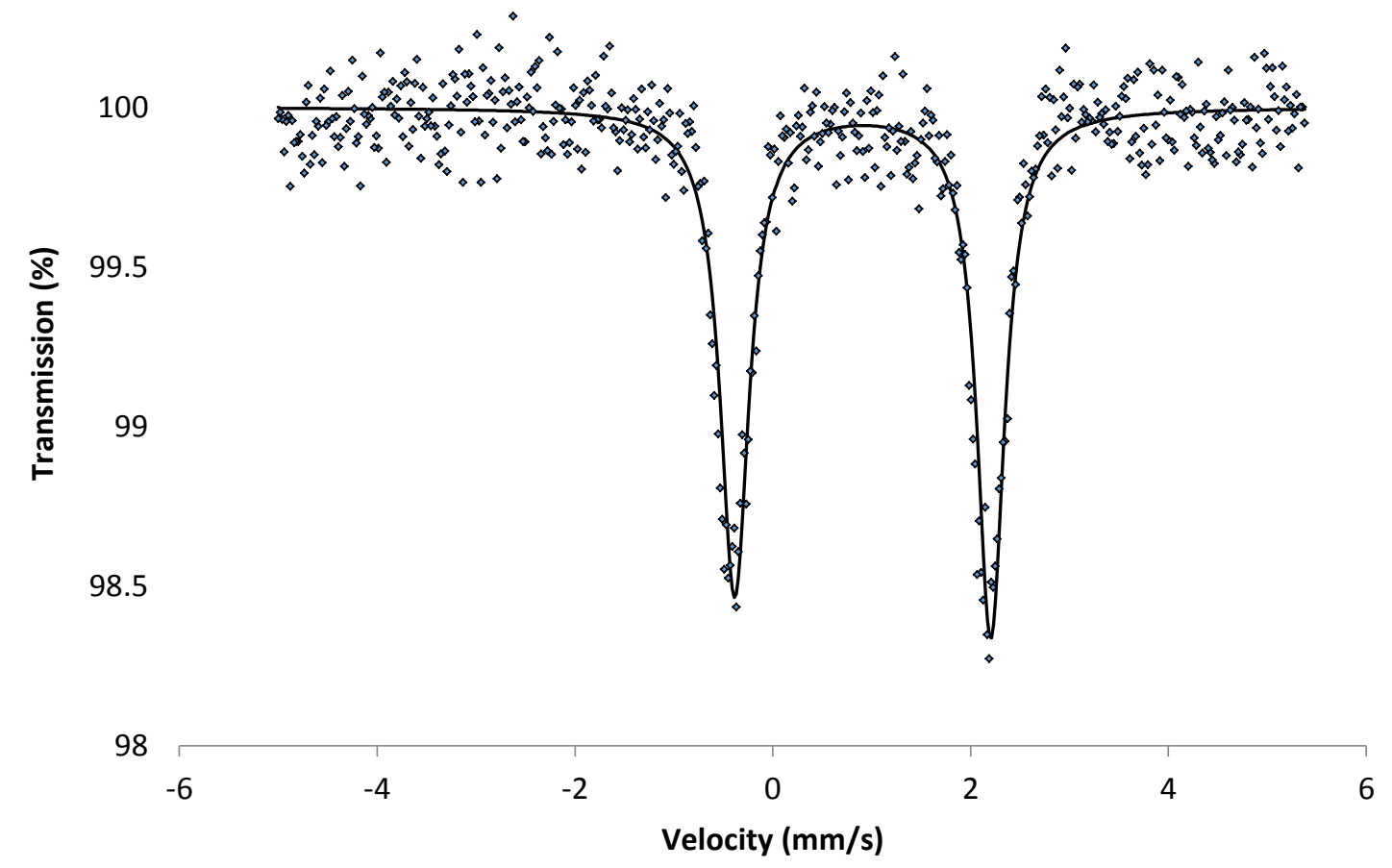

Figure S2. Mössbauer spectra of [Fe(7-Mes) $\left.\mathrm{Br}_{2}(\mathrm{THF})\right], 3$ recorded at $80 \mathrm{~K}$. 


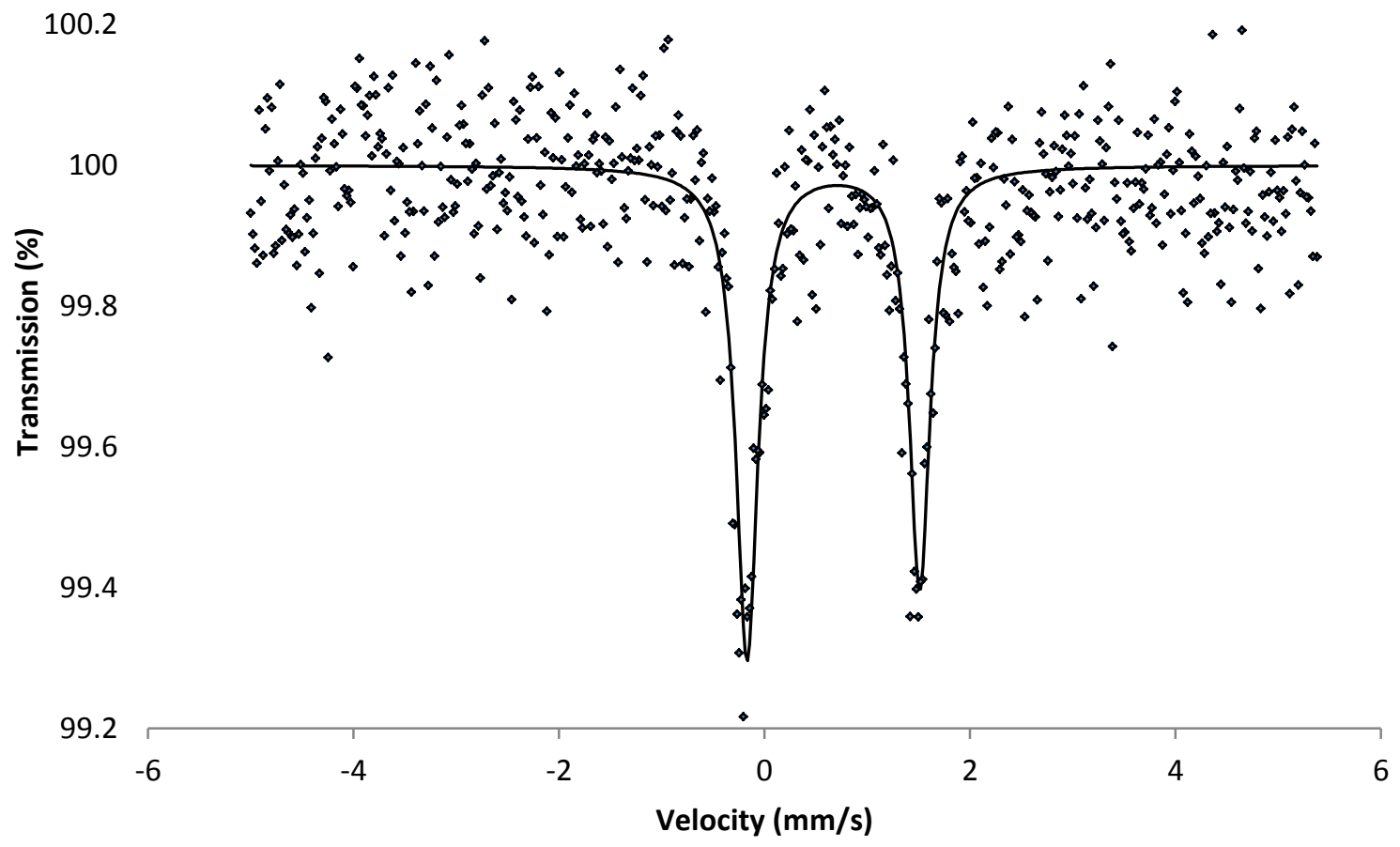

Figure S3. Mössbauer spectra of [Fe(7-Mes) $\left.\mathrm{Br}_{2}\right], 4$ recorded at $80 \mathrm{~K}$.

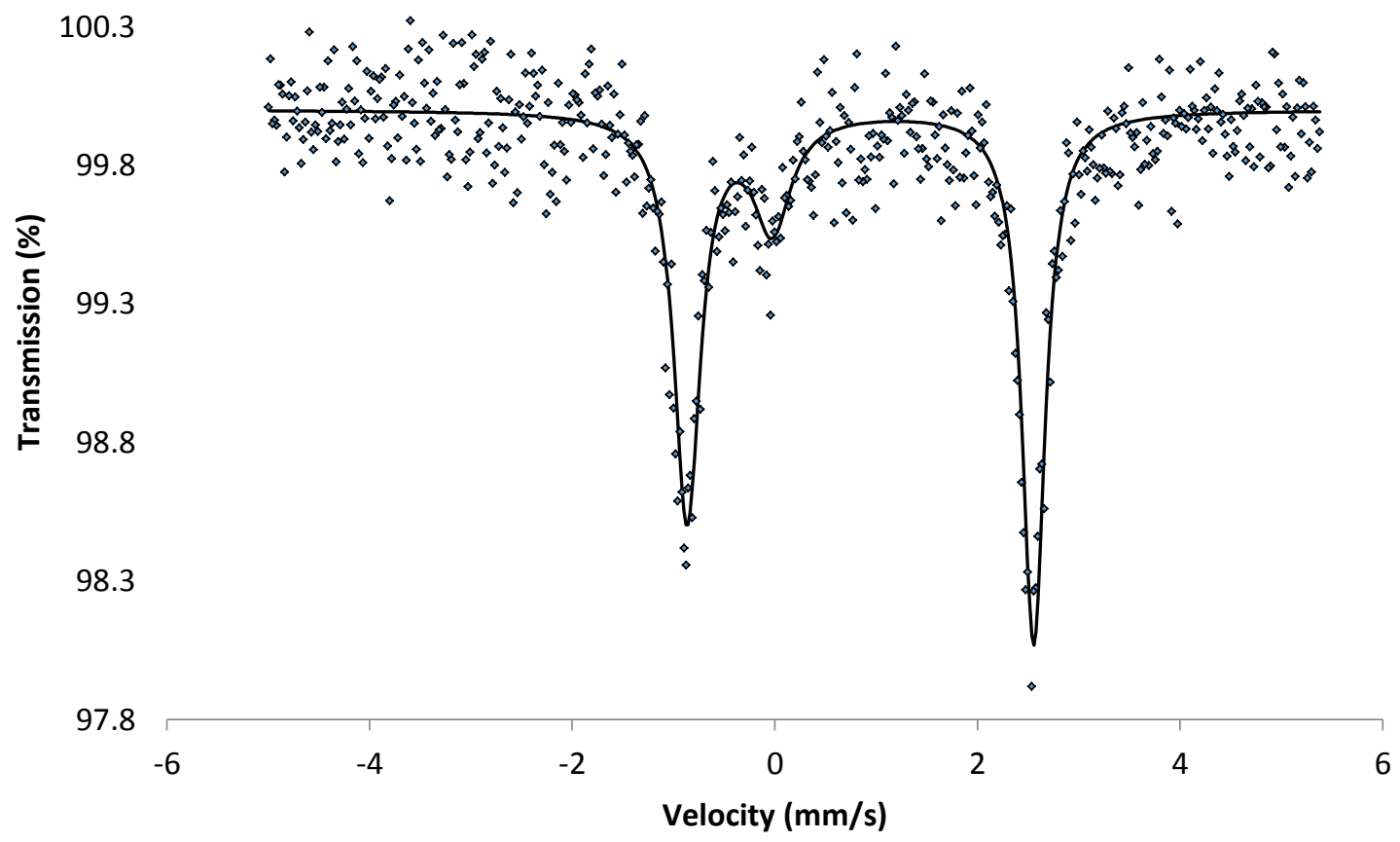

Figure S4. Mössbauer spectra of $\left[\mathrm{Fe}(\mathrm{SDiPP}) \mathrm{Br}_{2}\right]_{2}, \mathbf{5}$ recorded at $80 \mathrm{~K}$. This spectra was best modelled as two iron(II) species in $77 \%$ and $23 \%$ respectively. We postulate the major component $(77 \%)$ to be $\left[\mathrm{Fe}(\mathrm{SDiPP}) \mathrm{Br}_{2}\right]_{2}$. Multiple independently synthesised batches which returned satisfactory microanalyses always contained the same observed minor species. 


\section{SQUID Magnetometry Data}

The magnetic properties of polycrystalline samples of $\mathbf{1}$ and $\mathbf{3}$ were measured using a Quantum Design MPMS-7 SQUID magnetometer at temperatures in the range 1.8-300 K. The sample was prepared in a glove box by lightly crushing polycrystalline samples of $\mathbf{1}$ and 3 prior to transfer into an NMR tube, being restrained in eicosane (to prevent sample torquing) and flame sealed under vacuum.

\section{CASSSCF Calculation Information}

CASSCF calculations were performed with MOLCAS 8.0. ${ }^{[3]}$ The active space consisted of six electrons in the five $3 \mathrm{~d}$ orbitals. The $5 \mathrm{~S}=2,45 \mathrm{~S}=1$ and $50 \mathrm{~S}=0$ configurations were considered both in the orbital optimisation (RASSCF) and the spin-orbit mixing (RASSI) procedures, and the ZFS of the $\mathrm{S}=2$ ground state was extracted from the spin-orbit mixed states (SINGLE_ANISO). Given orbital energies are the energies of the five $\mathrm{S}=2$ states calculated in the CASSCF procedure. Basis sets from the ANO-RCC library of VTZP $(\mathrm{Fe})$, $\operatorname{VDZP}(\mathrm{Br}, \mathrm{C})$ and VDZ $(\mathrm{H})$ quality were employed, ${ }^{[4]}$ along with Cholesky decomposition of the two-electron integrals. All parameters took their default values. Matrix elements of orbital angular momentum between the spin-free states of the $S=2$ configurations taken from the output of the RASSI routine.

Structures for the deformation of torsion angles can be found in the accompanying .xyz file.

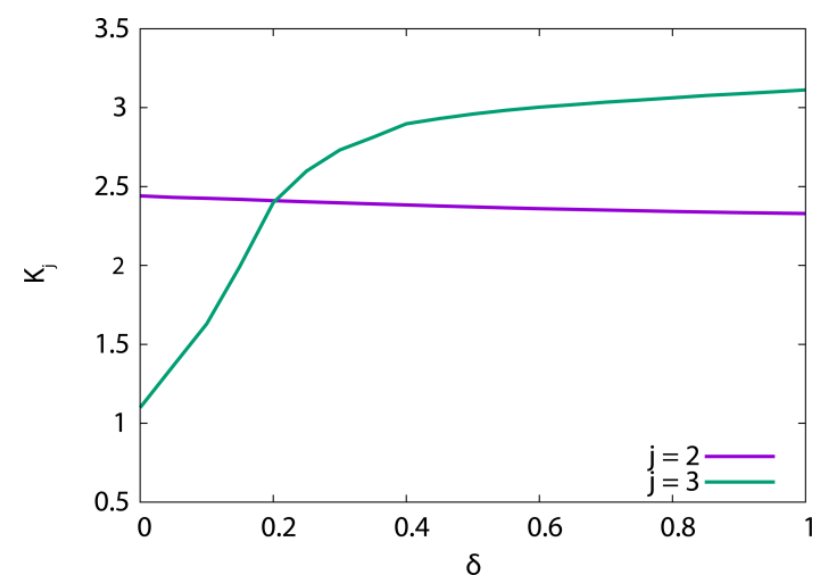

Figure S5. $\mathrm{K}_{\mathrm{j}}$ parameter for $\mathbf{4}$ as the bromide ions are deformed along the coordinate $\delta$.

$$
K_{j}=\sqrt{\sum_{\alpha, \beta \in x, y, z}\left(\left\langle\varphi_{1}\left|\hat{L}_{\alpha}\right| \varphi_{j}\right\rangle\left\langle\varphi_{j}\left|\hat{L}_{\beta}\right| \varphi_{1}\right\rangle\right)^{2}}
$$




\section{References}

[1] CrysAlisPro, Agilent Technologies, Version 1.171.35.19 (release 27-10-2011 CrysAlis171 .NET) (compiled Oct 27 2011,15:02:11)

[2] Olex2.refine (O. V. Dolomanov, L. J. Bourhis, R. J. Gildea, J. A. K. Howard and H. Puschmann, J. Appl. Cryst. (2009). 42, 339-341).

[3] Aquilante, F.; Autschbach, J.; Carlson, R. K.; Chibotaru, L. F.; Delcey, M. G.; De Vico, L.; Fdez. Galván, I.; Ferré, N.; Frutos, L. M.; Gagliardi, L.; Garavelli, M.; Giussani, A.; Hoyer, C. E.; Manni, G.; Lischka, H.; Ma, D.; Malmqvist, P. Å.; Müller, T.; Nenov, A.; Olivucci, M.; Pedersen, T. B.; Peng, D.; Plasser, F.; Pritchard, B.; Reiher, M.; Rivalta, I.; Schapiro, I.; Segarra-Martí, J.; Stenrup, M.; Truhlar, D. G.; Ungur, L.; Valentini, A.; Vancoillie, S.; Veryazov, V.; Vysotskiy, V. P.; Weingart, O.; Zapata, F.; Lindh, R. Journal of Computational Chemistry, 2015, DOI: 10.1002/jcc.24221.

[4] Roos, B. O.; Lindh, R.; Malmqvist, P-Å.; Veryazov, V.; Widmark, P.-O. J. Phys. Chem. A, 2005, 109, 6575-6579. 


\section{NMR Data for All Novel Compounds}

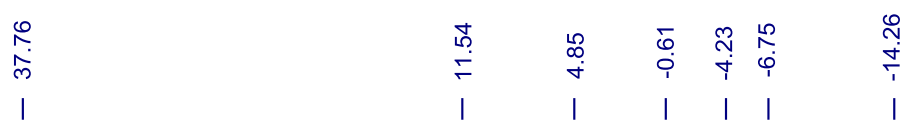

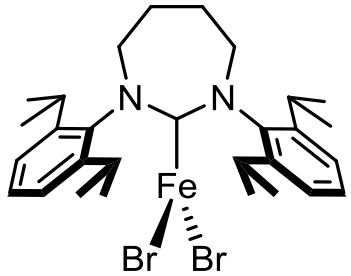

1

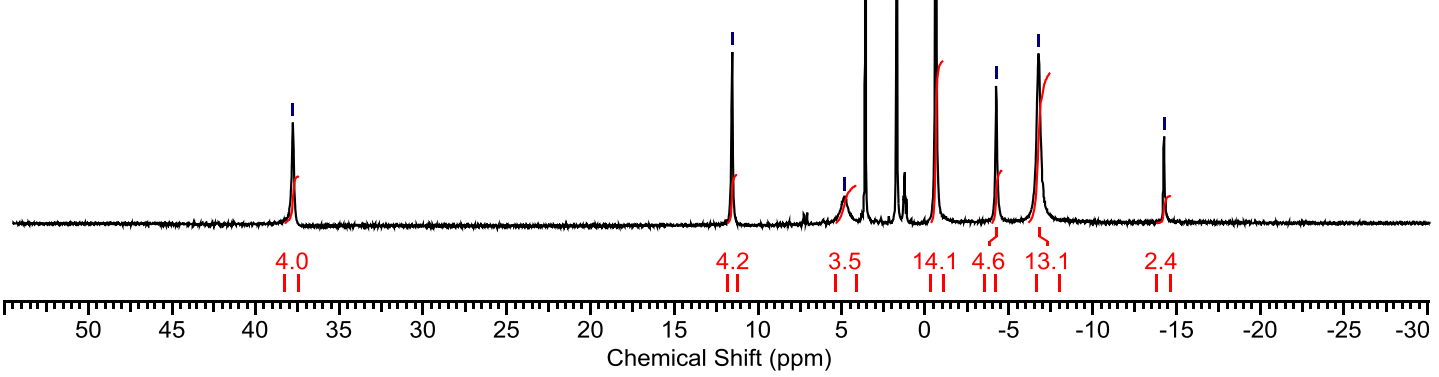

Figure S6. ${ }^{1} \mathrm{H}$ NMR of [Fe(7-DiPP)Br 2$], \mathbf{1}\left(\mathrm{THF}-d_{8}, 400 \mathrm{MHz}, 298 \mathrm{~K}\right)$.

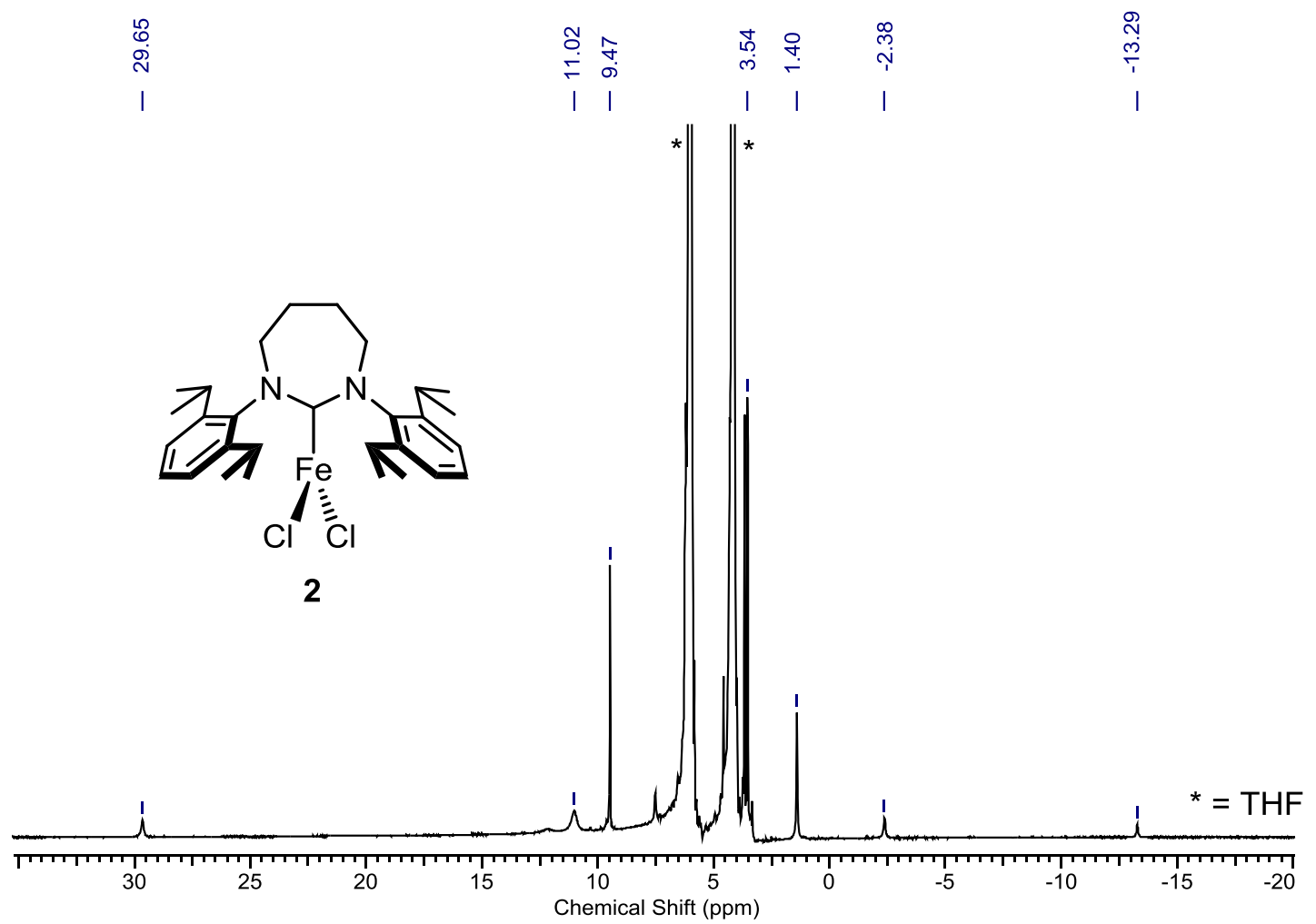

Figure S7. ${ }^{1} \mathrm{H}$ NMR of [Fe(7-DiPP)Cl 2 ], 2 (protio-THF, $400 \mathrm{MHz}, 298 \mathrm{~K}$ ). 


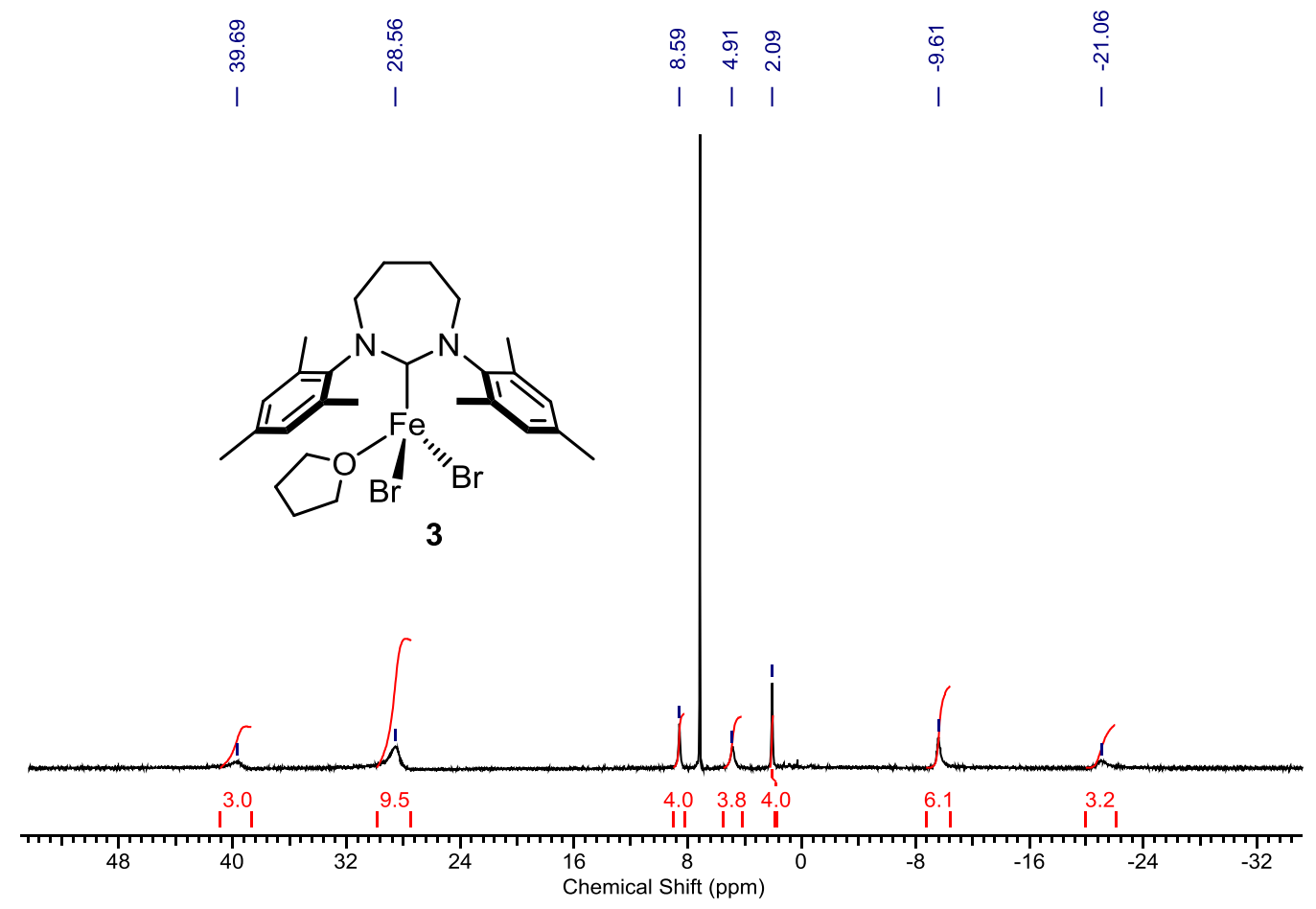

Figure S8. ${ }^{1} \mathrm{H}$ NMR of [Fe(7-Mes) $\left.\mathrm{Br}_{2}(\mathrm{THF})\right], 3\left(\mathrm{C}_{6} \mathrm{D}_{6}, 400 \mathrm{MHz}, 298 \mathrm{~K}\right)$.

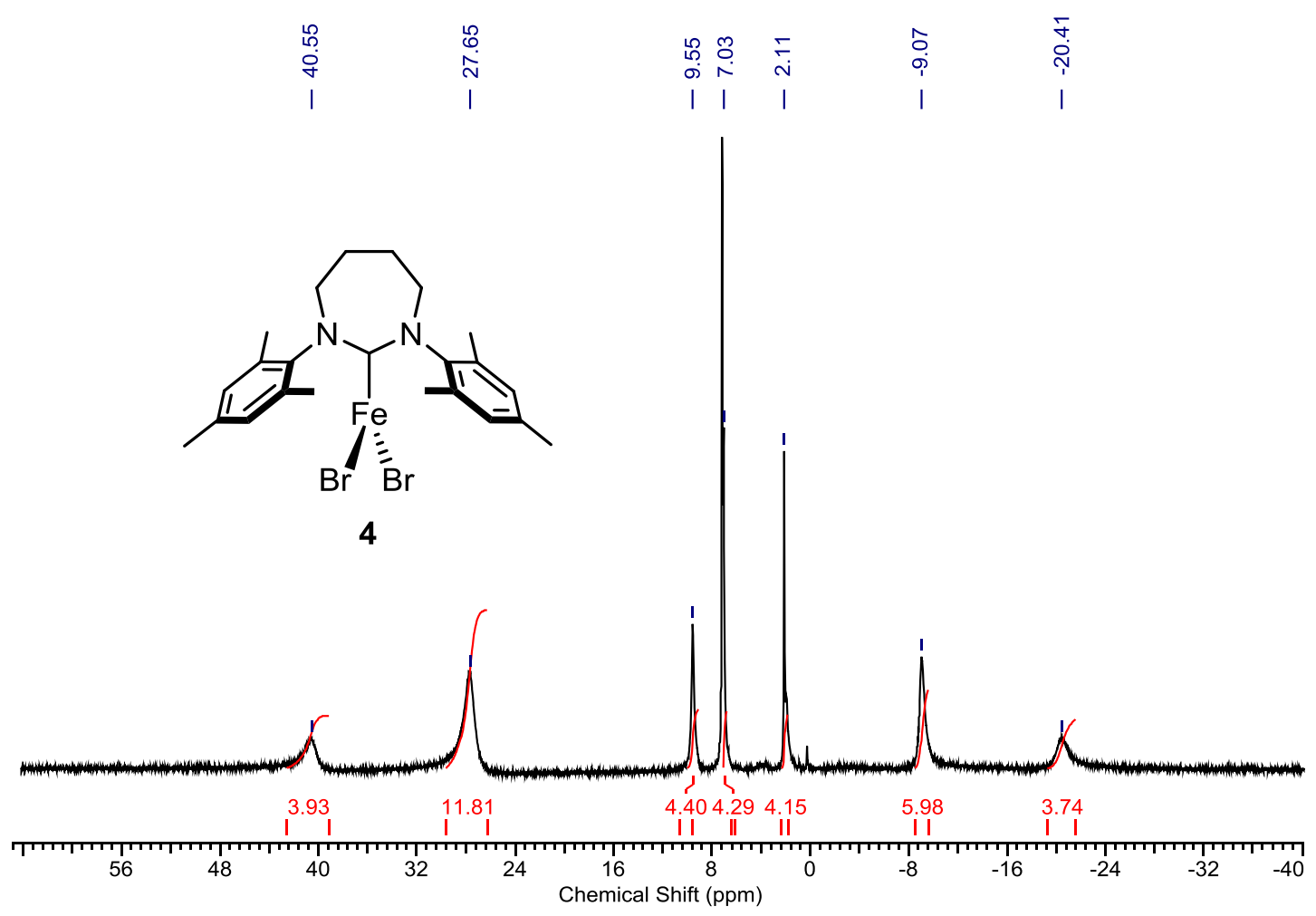

Figure S9. ${ }^{1} \mathrm{H} \mathrm{NMR}$ of $\left[\mathrm{Fe}(7-\mathrm{Mes}) \mathrm{Br}_{2}\right], 4\left(\mathrm{C}_{6} \mathrm{D}_{6}, 400 \mathrm{MHz}, 298 \mathrm{~K}\right)$. 


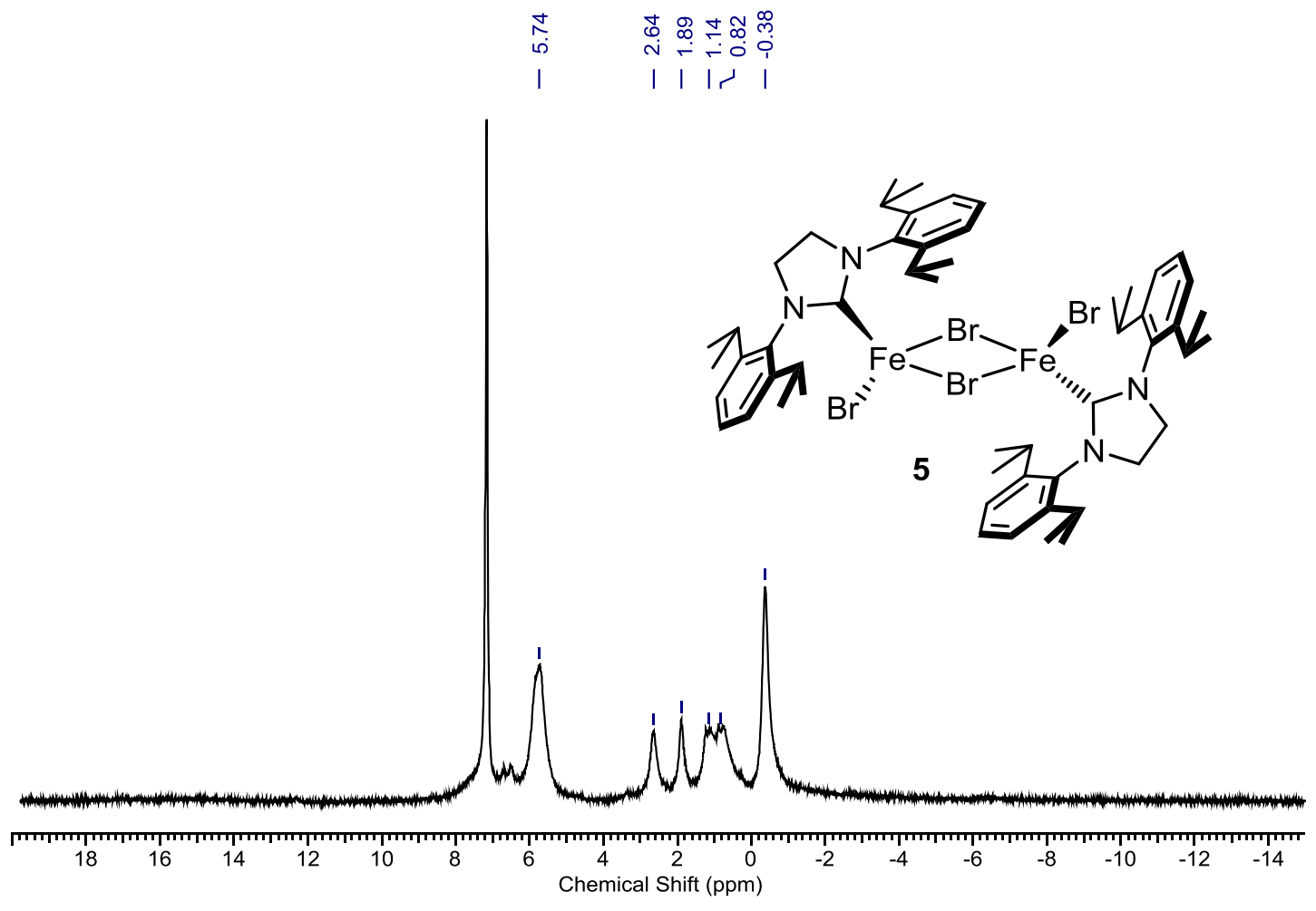

Figure S10. ${ }^{1} \mathrm{H} \mathrm{NMR}$ of $\left[\mathrm{Fe}(\mathrm{SDiPP}) \mathrm{Br}_{2}\right]_{2}, \mathbf{5}\left(\mathrm{C}_{6} \mathrm{D}_{6}, 400 \mathrm{MHz}, 298 \mathrm{~K}\right)$.

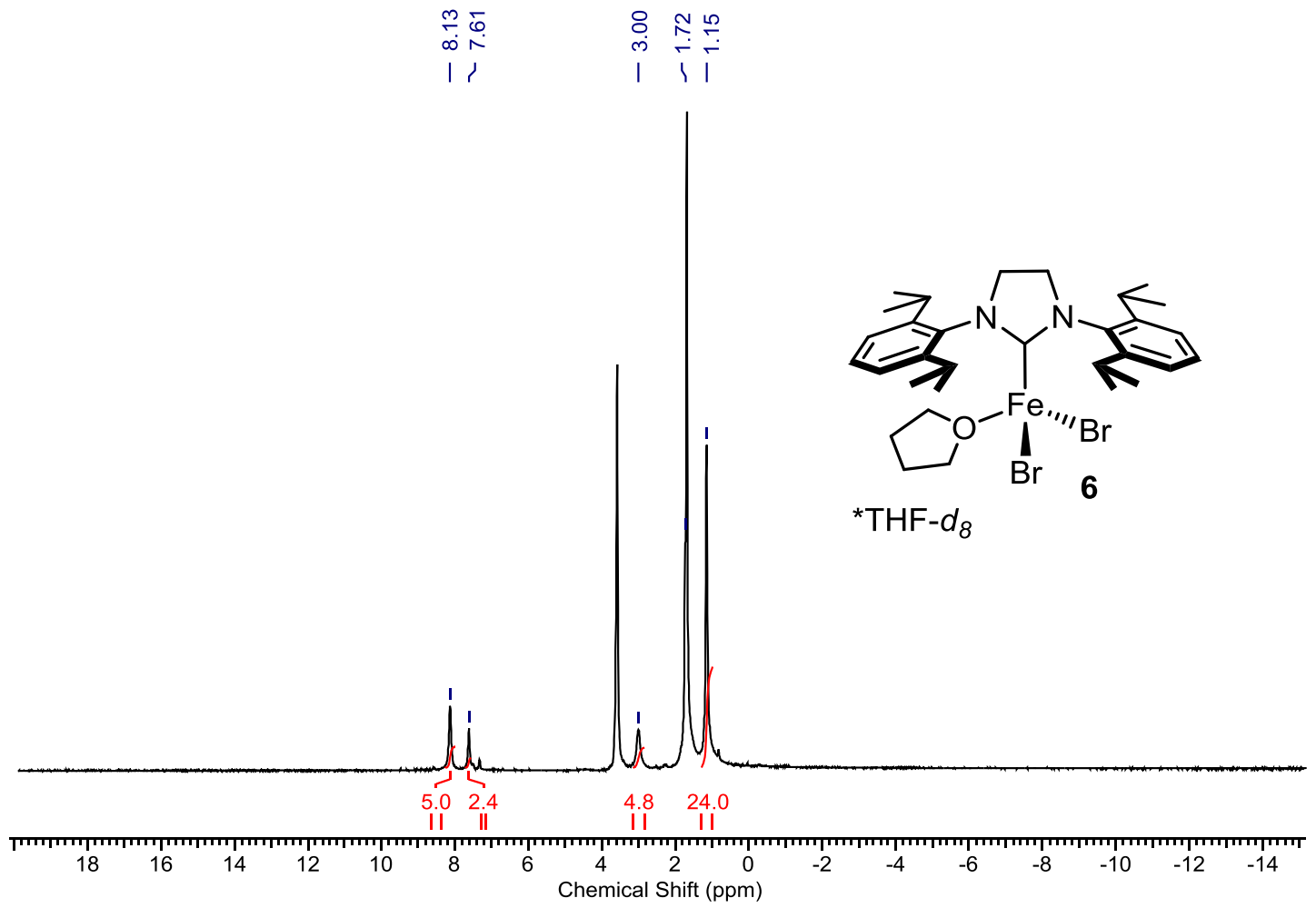

Figure S11. ${ }^{1} \mathrm{H}$ NMR of [Fe(SDiPP)Br $\left.2\left(\mathrm{THF}-d_{8}\right)\right], 6$ (THF- $\left.d_{8}, 400 \mathrm{MHz}, 298 \mathrm{~K}\right)$. 


\section{Additional NMR Experiments}

6a. The addition of excess $d_{8}$-THF to a $\mathrm{C}_{6} \mathrm{D}_{6}$ solution of [Fe(7-DiPP)Br 2$], 1$

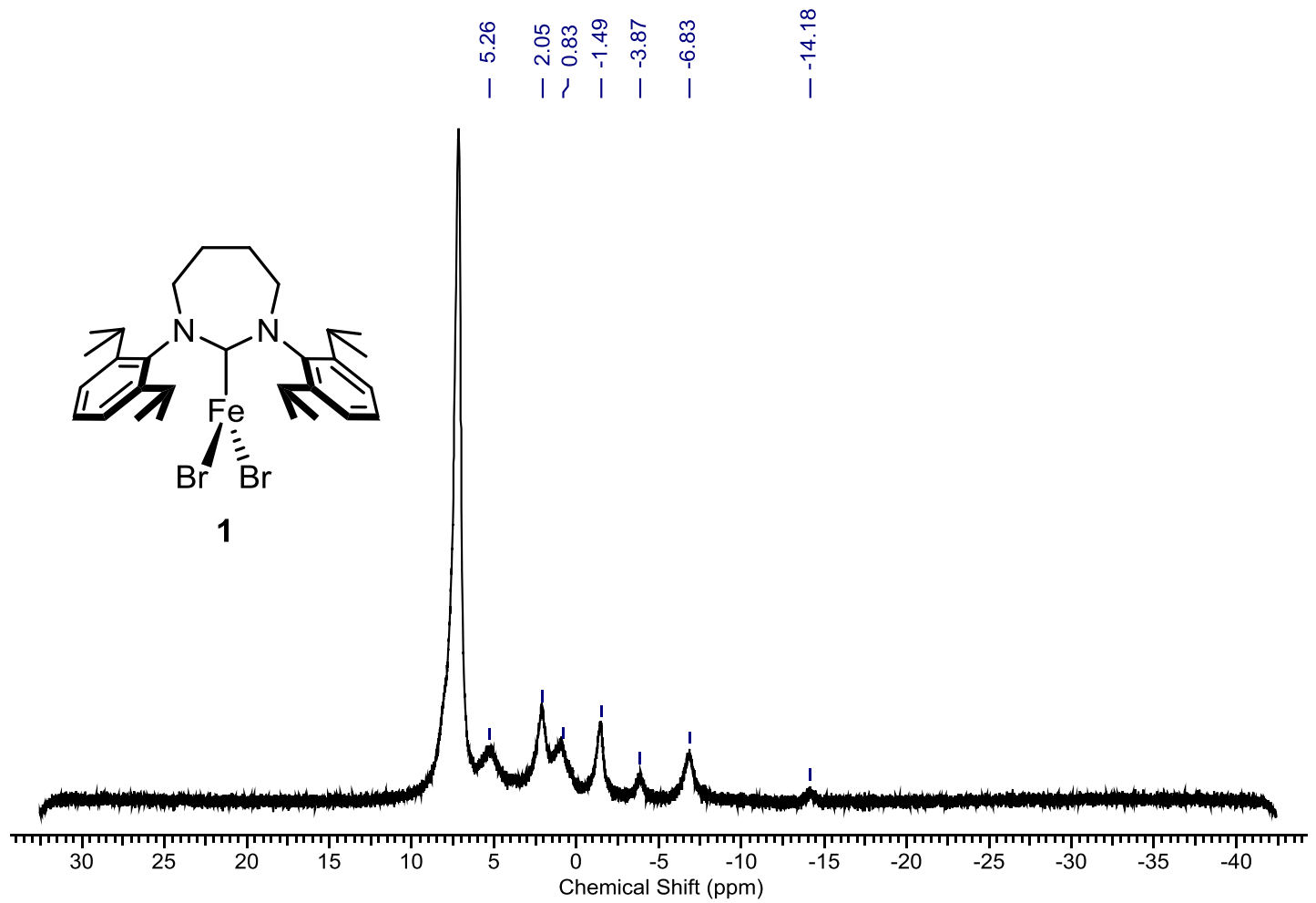

Figure S12. ${ }^{1} \mathrm{H}$ NMR $\left(\mathrm{C}_{6} \mathrm{D}_{6}, 400 \mathrm{MHz}, 298 \mathrm{~K}\right)$ of: [Fe(7-DiPP $\left.) \mathrm{Br}_{2}\right], \mathbf{1}$ in $\mathrm{C}_{6} \mathrm{D}_{6}$.

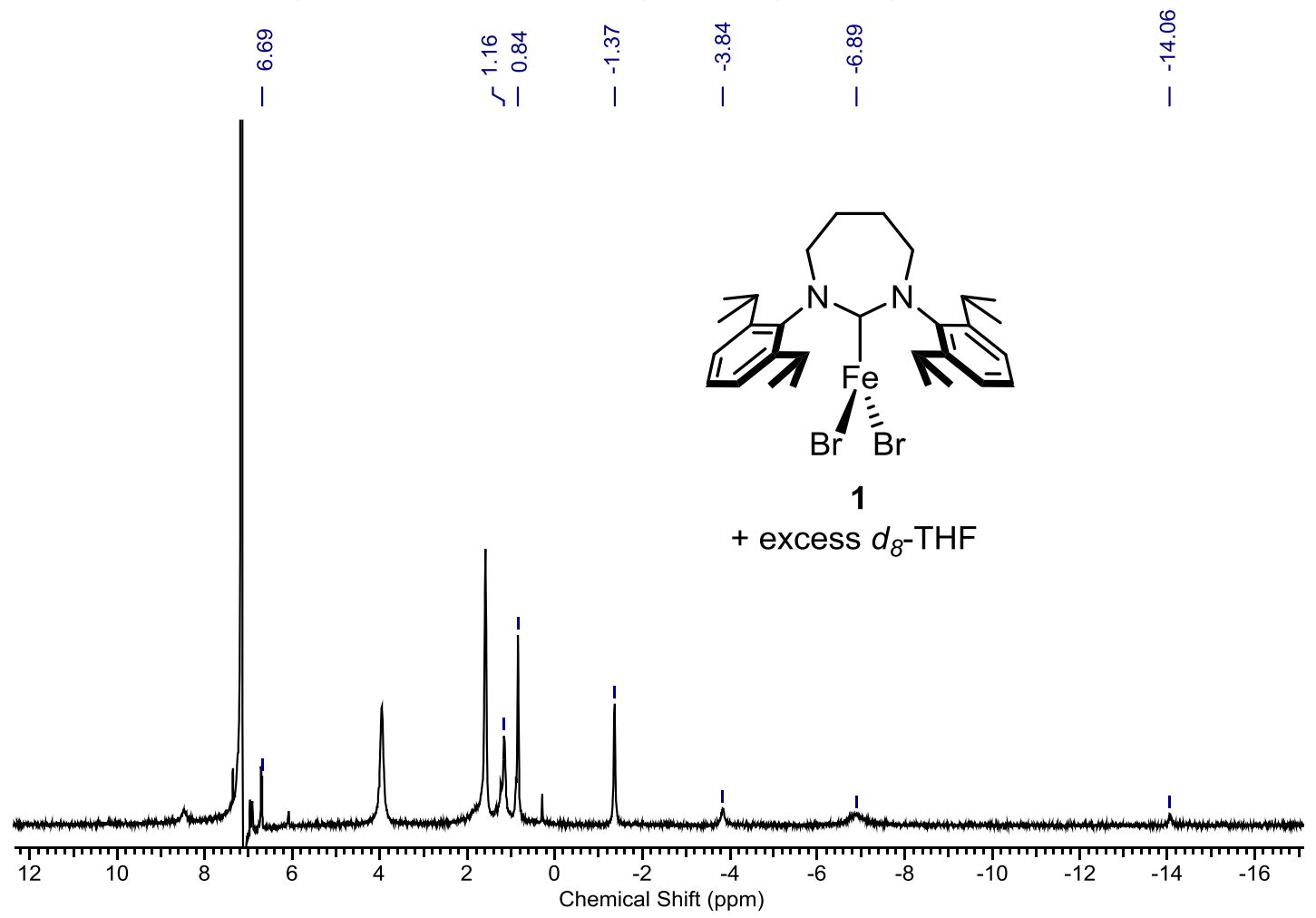

Figure S13. ${ }^{1} \mathrm{H}$ NMR $\left(\mathrm{C}_{6} \mathrm{D}_{6}, 400 \mathrm{MHz}, 298 \mathrm{~K}\right)$ of: [Fe(7-DiPP)Br ${ }_{2}$ ], 1 in $\mathrm{C}_{6} \mathrm{D}_{6}$ with an excess of $d_{8}$-THF. 
6b. The addition of excess $d_{8}$-THF to a $\mathrm{C}_{6} \mathrm{D}_{6}$ solution of [ $\left.\mathrm{Fe}(7-\mathrm{Mes}) \mathrm{Br}_{2}\right], 4$

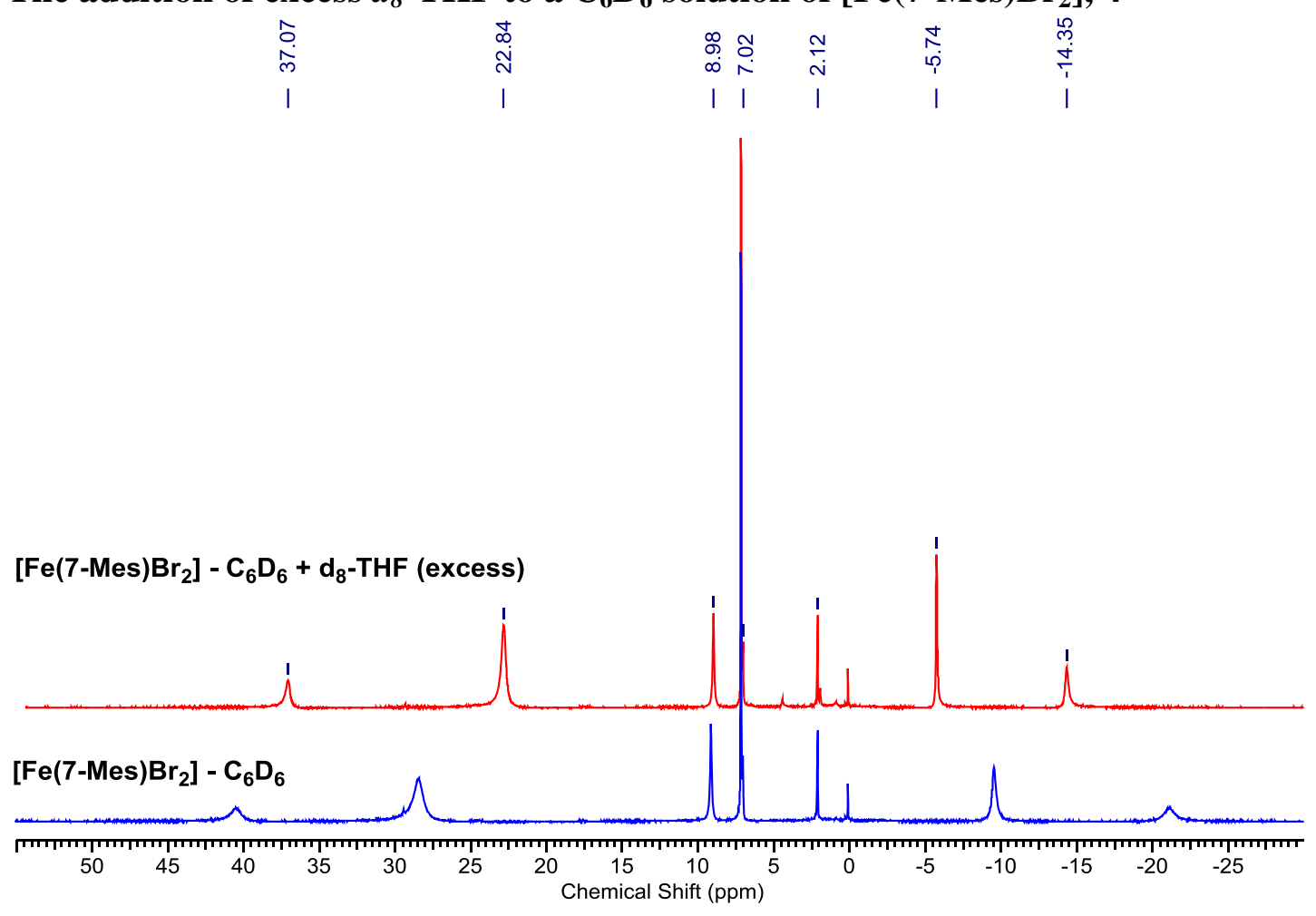

Figure S14. Collected ${ }^{1} \mathrm{H}$ NMR $\left(\mathrm{C}_{6} \mathrm{D}_{6}, 400 \mathrm{MHz}, 298 \mathrm{~K}\right)$ of: [Fe(7-Mes) $\mathrm{Br}_{2}$ ], 4 (blue); and [Fe(7-Mes)Br $\mathrm{Br}_{2}$, 4 spiked with excess $d_{8}$-THF (red). 